\title{
Tendance à plus de douceur dans les vins : comment les consommateurs apprécient-ils les vins avec léger sucre résiduel ?
}

\author{
P. Deneulin ${ }^{1}$, J. Boven ${ }^{1}$, C. Bourcet ${ }^{2}$ et M.-H. Corajod ${ }^{2}$ \\ ${ }^{1}$ Changins, Viticulture and Oenology, HES-SO University of Applied Sciences and Arts, Western Switzerland, Route de Duillier 50, \\ 1260 Nyon, Suisse \\ 2 BFH-HAFL, Applied University of Agricultural, Forest and Food Science, Länggasse 85, 3052 Zollikofen, Suisse
}

\begin{abstract}
Résumé. La tendance à des vins plus sucrés tend à se généraliser à l'échelle mondiale tant par le réchauffement climatique que par le changement de goût des consommateurs. Cette étude vise à recueillir l'appréciation des consommateurs suisses envers quatre vins blancs et quatre vins rouges de profils sensoriels variés, avec ou sans sucre résiduel. Les vins avec 6 à $9 \mathrm{~g} / \mathrm{L}$ de sucres résiduels, qu'ils soient blancs ou rouges, sont globalement préférés des consommateurs. Toutefois et au-delà de cette préférence, il est possible d'identifier des sous-groupes de consommateurs aux préférences variables. Les jeunes femmes semblent également apprécier les vins blancs moins sucrés s'ils sont fruités. Au contraire, les personnes les plus âgées semblent se tourner vers les vins boisés ou aux arômes évolués.
\end{abstract}

\section{Introduction}

L'attirance pour le goût sucré est une caractéristique innée. Dès le plus jeune âge, les nourrissons montrent une réaction positive au goût sucré, signe d'un aliment riche, comme peut l'être le lait maternel [1]. A l'opposé, l'acceptation du goût amer, biologiquement relié à des plantes toxiques, nécessite un apprentissage au cours de la vie. Toutefois, c'est vraiment au cours des dernières décennies, et plus particulièrement ces cinquante dernières années, que la consommation de sucre a significativement augmenté. Ce phénomène engendre de réels problèmes de santé publique avec une forte hausse de l'obésité et du diabète. Aujourd'hui, le sucre est considéré comme un produit addictif au même titre que certaines drogues, engendrant des phénomènes de dépendance [2].

Le niveau d'expérience semble être un critère important en termes de segmentation des consommateurs par rapport à leur appréciation des vins sucrés. Francis et Williamson (2015) [3] montrent que les vins secs sont moins bien appréciés des nouveaux consommateurs australiens. Les vins sucrés apparaissent donc comme une possibilité pour ces consommateurs nouvellement intéressés par le vin, sans que ceux-ci ne soient nécessairement jeunes en termes d'âge. Dès les années 50 , les consommateurs américains avaient une préférence nette pour les vins sucrés [4]. Amerine et Ough (1967) [5] démontrent qu'aux Etats Unis, où les rosés sont souvent associés à de la sucrosité, même les consommateurs américains expérimentés préfèrent les vins rosés contenant entre 10 et $20 \mathrm{~g} / \mathrm{L}$ de sucre. Plus récemment, Blackman et al. (2010) [6] ont étudié les préférences de consommateurs (expérimentés et novices) et de producteurs pour des Sémillons dans lesquels ils ont ajoutés de 2 à $32 \mathrm{~g} / \mathrm{L}$ de glucose. Si les novices préfèrent des vins plus sucrés que les consommateurs expérimentés, l'acceptabilité des professionnels diminue avec le taux de sucre.

L'Europe n'est pas épargnée par cette tendance et l'augmentation du sucre dans les vins a déjà été mise en évidence par Pitte en 2007 [7]. L'uniformisation du goût des consommateurs se retrouve également pour le vin où, même si les notions de typicité régionale semblent être importantes aux yeux des consommateurs, leurs préférences organoleptiques se tournent majoritairement vers les cépages internationaux [8]. La nouvelle tendance de vins plus faciles avec du sucre résiduel semble donc mondiale.

L'objectif de cet article est d'étudier l'acceptabilité de plusieurs styles de vins blancs et rouges, avec ou sans sucres résiduels, par les consommateurs suisses.

\section{Matériel et méthodes}

\subsection{Les vins}

Quatre vins blancs et quatre vins rouges du commerce ont été sélectionnés pour représenter la diversité présente sur le marché suisse. Pour chaque série, il a été choisi d'avoir un vin avec un léger sucre résiduel, un vin boisé et deux vins plus ou moins aromatiques.

Parmi les quatre vins blancs (Tableau 1), le Pinot Gris et le Chardonnay contenaient autour de $6 \mathrm{~g} / \mathrm{L}$ de sucre avec un profil fruité pour le premier et boisé pour le second. La Petite Arvine correspond à un vin sec et fruité caractéristique de la région du Valais. Le Chasselas choisi était du millésime 1993 permettant d'étudier l'acceptabilité des consommateurs pour des vins au profil plus évolué.

Pour les vins rouges, trois cépages internationaux et un assemblage ont été choisis (Tableau 2). Le Gamay et le Merlot étaient deux vins secs aux profils aromatiques 
Tableau 1. Liste des quatre vins blancs et caractéristiques analytiques (sucre et $\mathrm{pH}$ ).

\begin{tabular}{|l|c|c|c|}
\hline Cépage & Millésime & Sucre $(\mathrm{g} / \mathrm{L})$ & $\mathrm{pH}$ \\
\hline Chasselas & 1993 & 0.00 & 3.42 \\
\hline Pinot Gris & 2016 & 6.60 & 3.51 \\
\hline Petite Arvine & 2016 & 0.00 & 3.47 \\
\hline Chardonnay & 2015 & 6.30 & 3.31 \\
\hline
\end{tabular}

Tableau 2. Liste des quatre vins rouges et caractéristiques analytiques (sucre et $\mathrm{pH}$ ).

\begin{tabular}{|c|c|c|c|}
\hline Cépage & Millésime & Sucre $(\mathrm{g} / \mathrm{L})$ & $\mathrm{pH}$ \\
\hline Gamay & 2016 & 0.00 & 3.38 \\
\hline Merlot & 2016 & 0.00 & 3.46 \\
\hline Pinot Noir & 2015 & 3.79 & 3.58 \\
\hline Assemblage & 2016 & 9.19 & 3.52 \\
\hline
\end{tabular}

variés. Le Pinot noir était un vin boisé et l'assemblage rouge un rouge technique édulcoré par moût concentré rectifié et tanisé en fin de vinification. Ces deux vins contenaient respectivement 3.8 et plus de $9.2 \mathrm{~g} / \mathrm{L}$ de sucre résiduel.

\subsection{Les consommateurs}

224 consommateurs suisses ont participé à l'étude dont $54 \%$ d'hommes et $46 \%$ de femmes. Tous les dégustateurs étaient des consommateurs réguliers de vins avec seulement $15 \%$ ne consommant pas hebdomadairement du vin et $12.5 \%$ disant en consommer quotidiennement, les autres en consommant de une à plusieurs fois par semaine.

$58 \%$ ont déclaré avoir un niveau de connaissance en vin de faible à très faible, $28 \%$ moyen et $14 \%$ bon. Toutes les tranches d'âge étaient représentées avec $17 \%$ de $18-$ 29 ans, $11 \%$ de 30-44 ans, 35\% de 45-59 ans, $29 \%$ de $60-74$ ans et $8 \%$ de plus de 74 ans.

\subsection{Protocole expérimental}

Un panel expert a préalablement établi les caractéristiques sensorielles de chacun des vins au moyen d'un profil sensoriel composé de 29 descripteurs pour les vins blancs et 25 descripteurs pour les vins rouges.

Les tests consommateurs ont eu lieu dans des cabines d'analyse sensorielle situées dans deux régions de Suisse (Nyon et Berne). Pour chacun des vins, les consommateurs devaient donner leur appréciation hédonique sur une échelle structurée en 9 points allant de 1 (très mauvais) à 9 (excellent). Ils pouvaient également ajouter des commentaires sur ce qui leur avait plu ou déplu puis cocher l'ensemble des attributs caractérisant le vin selon eux parmi une liste de 24 termes descriptifs proposés (méthode Check-All-That-Apply).

Tous les vins ont été servis dans des verres INAO transparents à raison de $20 \mathrm{ml}$, anonymisés par des codes à trois chiffres et servis de manière monadique selon un plan en carré latin de Williams. Les consommateurs ont évalué les quatre vins blancs suivis des quatre vins rouges.

A la fin de la dégustation, chaque participant a dû remplir un formulaire contenant 13 questions sur

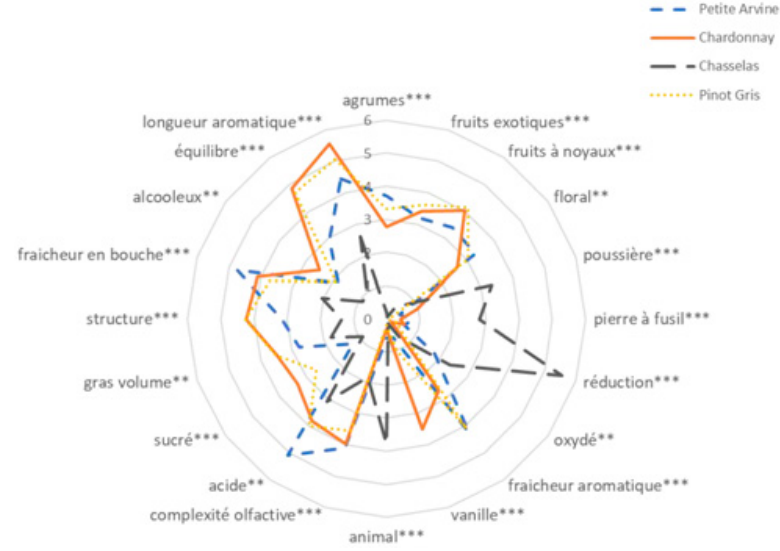

Figure 1. Profil sensoriel des 4 vins blancs (** descripteurs significatifs à $5 \%$ et $* * *$ à $1 \%$ ).

ses habitudes de consommation. Les données ont été entièrement saisies sur ordinateur au moyen du logiciel Fizz (Biosystèmes).

\subsection{Analyses statistiques}

Le profil sensoriel a été analysé par des analyses de variance à 2 facteurs (vin et juge). Les notes d'acceptabilité des consommateurs ont également été analysées par analyse de variance à 2 facteurs. La segmentation des consommateurs en fonction de leurs préférences a été réalisée au moyen d'une classification k-means. L'ensemble des analyses ont été réalisées avec le logiciel $\mathrm{R}$ implémenté des packages SensoMineR et FactoMineR et le logiciel Minitab.

\section{Résultats}

\subsection{Profil sensoriel des vins}

Parmi les 29 descripteurs évalués pour décrire les vins blancs, 20 permettent de différencier les vins à un seuil de significativité de $5 \%$ (Fig. 1).

La Petite Arvine est caractérisée par ses odeurs d'agrumes, sa fraicheur aromatique et en bouche et son acidité plus élevée. Le Chasselas est typique d'un vin évolué avec des odeurs d'oxydation couplées à de la réduction et des odeurs de pierre à fusil caractéristiques des vins bouchés avec une capsule à vis. Le Chardonnay et le Pinot Gris suivent un profil assez proche avec du sucre, mais aussi de la structure et de l'équilibre en bouche et une plus longue persistance aromatique. Le Chardonnay se distingue par les notes de vanille apportées par le passage en barrique alors que le Pinot Gris a plus de fraicheur aromatique.

Parmi les 25 descripteurs évalués pour les vins rouges, seuls 5 sont significatifs à $5 \%$ et 4 de plus à $10 \%$ (Fig. 2). Le Gamay a été considéré comme acide et montrant des odeurs de réduction. Le Merlot est apparu comme le plus intense au nez. Le Pinot Noir était assez caractéristique avec des odeurs de fruits noirs et de la vanille apportée par un élevage barrique. Enfin, l'assemblage s'est surtout distingué par une saveur sucrée plus intense. 


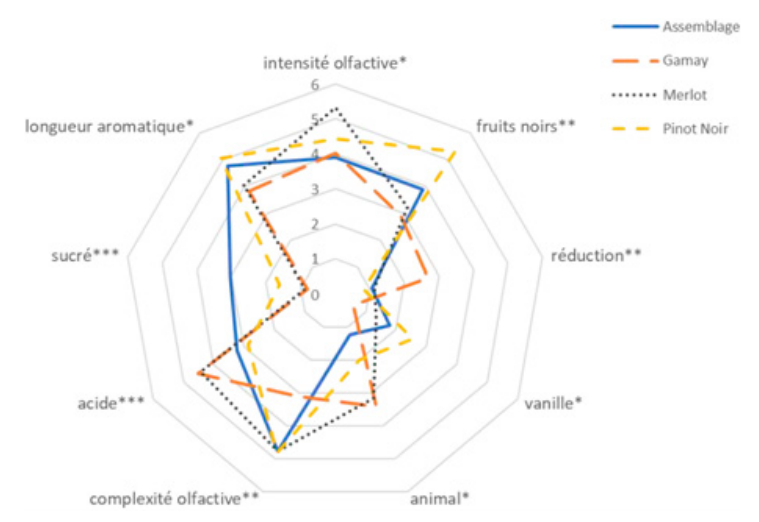

Figure 2. Profil sensoriel des 4 vins rouges (* descripteurs significatifs à $10 *$, ** à $5 \%$ et *** à $1 \%$ ).

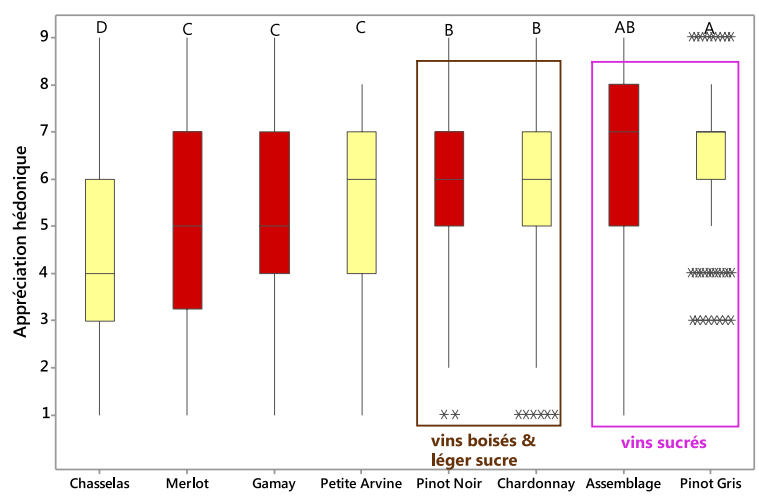

Figure 3. Boites à moustaches illustrant la diversité des notes hédoniques attribuées par les 224 consommateurs. Les lettres correspondent au résultat du test de Tukey, deux vins ne partageant aucune lettre en commun sont significativement différents en termes d'appréciation hédonique.

\subsection{Appréciation hédonique globale}

L'analyse de la variance montre que les vins n'ont pas été appréciés de manière identique $\left(\mathrm{F}_{(7,1561)}=49.2\right.$, $p$-valeur $<0.001)$. Le Pinot Gris recueille un consensus fort de la part des consommateurs avec $50 \%$ de notes à 6 ou 7 et personne ne l'ayant jugé en dessous d'une note de 3 (Fig. 3). Les quatre vins présentant un sucre résiduel ont été globalement préférés et jugés de manière plus consensuelle. A l'opposé, le Chasselas 1993 n'a pas convaincu les consommateurs avec une moyenne de 4.1.

La Fig. 4 illustre très clairement le lien entre la sucrosité perçue des vins et les moyennes d'appréciation hédonique. Ainsi, dans l'intervalle de perception sucrée allant de 0.5 à 3.5 (sur une échelle de 10) correspondant ici de 0 à $9 \mathrm{~g} / \mathrm{L}$ de sucre résiduel, plus la perception sucrée croit, plus la préférence des consommateurs augmente avec un $r^{2}$ allant jusqu'à 0.9 lorsque l'on enlève le Chasselas évolué du calcul. Il est intéressant de souligner que l'Assemblage rouge a été perçu comme moins intense en termes de sucrosité alors que sa concentration en sucres résiduels est plus élevée. La structure tannique de ce vin contrebalance la sucrosité afin de lui donner plus d'équilibre.

\subsection{Caractérisation des vins par attributs}

Après avoir noté leur appréciation hédonique, les consommateurs avaient la possibilité de cocher les attributs

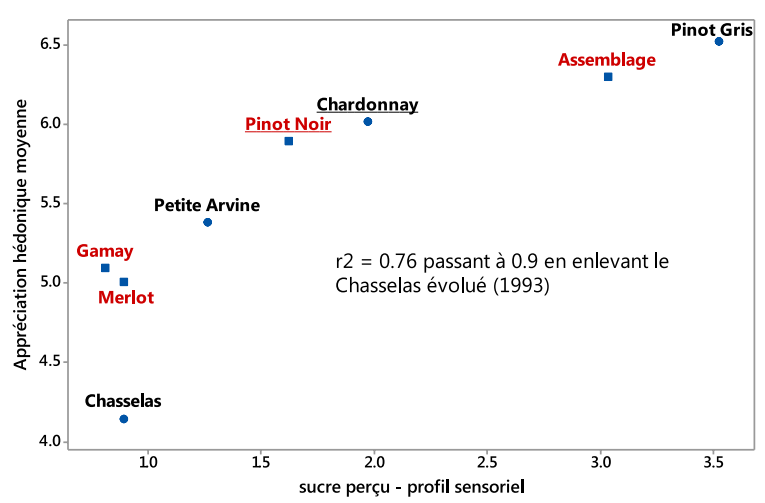

Figure 4. Nuage de points reliant les niveaux d'intensité de la saveur sucrée évaluée par le panel expert et les moyennes des appréciations hédoniques des 224 consommateurs. Les deux vins boisés ont été soulignés.

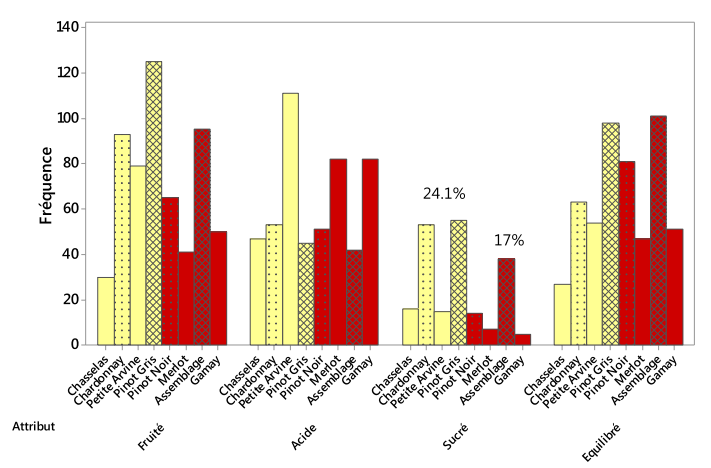

Figure 5. Fréquence pour laquelle chacun des quatre termes fruité, acide, sucré et équilibré ont été cochés par les 224 consommateurs (en pointillés les vins boisés et sucrés et en quadrillés les vins sucrés).

caractérisant le vin parmi une liste de 24 termes sensoriels proposés (méthode CATA). Parmi ces termes, quatre méritent une attention particulière (Fig. 5). Hormis pour le Chasselas 1993, les trois vins les moins appréciés ont été considérés comme acides et très peu sucrés par les consommateurs. Le Pinot Gris, l'Assemblage rouge et le Chardonnay ont été les plus fréquemment associés au terme fruité. Il est à noter que les trois vins avec 6 à $9 \mathrm{~g} / \mathrm{L}$ de sucre résiduel ont été ceux le plus fréquemment associés au terme sucré. Toutefois, cela ne représente que de 17 à $24 \%$ des consommateurs. La sucrosité de ces trois vins n'a donc pas été évidente pour une large majorité des consommateurs, mais ces vins leur sont apparus comme moins acides et surtout plus équilibrés. Le Pinot Noir, avec $3.8 \mathrm{~g} / \mathrm{L}$ de sucre n'a pas été considéré comme sucré par les consommateurs, mais plutôt comme équilibré.

\subsection{Segmentation des consommateurs}

\subsubsection{Vins blancs}

Une segmentation des consommateurs en fonction de leurs appréciations hédoniques (méthode des k-means) a permis de faire ressortir trois groupes distincts. La Fig. 6 montre que si les préférences sont variables sur trois des vins, tous les groupes ont une moyenne entre 6.3 et 6.8 pour le Pinot Gris. Celui-ci fait l'unanimité et reste, quels que soit les consommateurs, un vin particulièrement apprécié.

Le groupe 1 est composé de $28 \%$ des consommateurs et regroupe ceux qui rejettent le Chardonnay (moyenne 


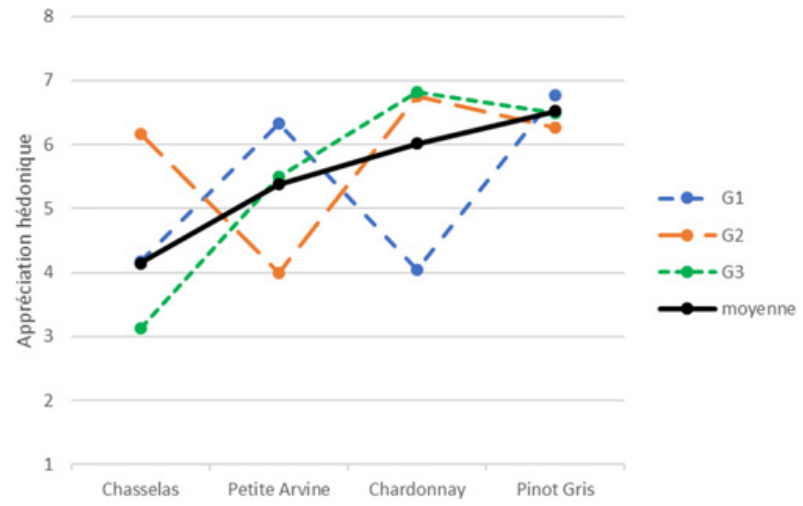

Figure 6. Comparaison des moyennes d'appréciation hédonique des quatre vins blancs pour l'ensemble des 224 consommateurs (trait plein) et pour chacun des trois groupes issus de la segmentation (traits pointillés).

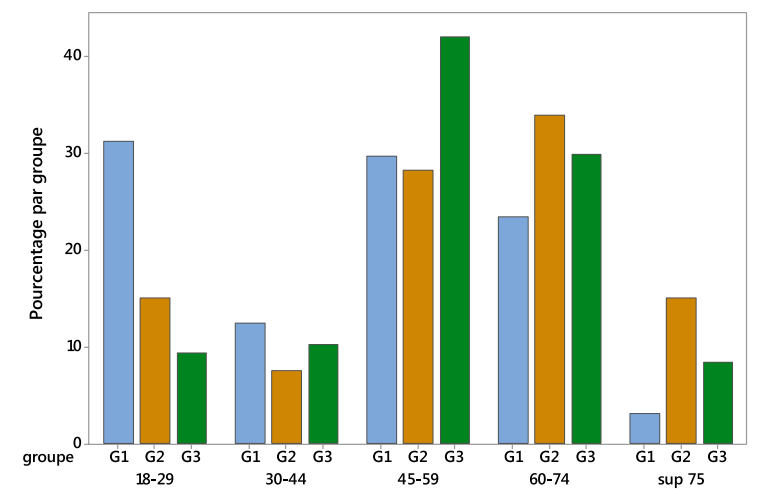

Figure 7. Comparaison des tranches d'âges en fonction des groupes de consommateurs. Les pourcentages ont été rapportés au nombre de personnes par groupe.

de 4.05 contre 6.01 pour les 224 consommateurs) mais apprécient plus que les autres la Petite Arvine (moyenne de 6.33 contre 5.38). Leurs préférences semblent donc se diriger vers des vins fruités, quelles que soient l'acidité et la sucrosité perçues.

Le groupe 2 est composé de $24 \%$ des consommateurs. Il s'oppose totalement au précédent en termes de préférences sensorielles avec une forte attractivité pour des vins aux arômes boisés (Chardonnay avec une moyenne 6.75 contre 6.01) ou évolués (Chasselas avec une moyenne de 6.17 contre 4.14). A l'opposé, ils rejettent la Petite Arvine (moyenne de 4 contre 5.38).

Enfin, le $3^{\text {ème }}$ groupe $(48 \%$ ) correspond aux personnes axant clairement leurs préférences sur les vins avec du sucre résiduel, le Chardonnay (6.82) et le Pinot Gris (6.52) et rejetant très fortement le Chasselas évolué (3.12).

L'âge apparait comme l'un des facteurs permettant de segmenter les préférences des consommateurs. En effet, le premier groupe est composé de $31 \%$ de jeunes de 18 à 29 ans contre $17 \%$ de personnes de cette tranche d'âge sur l'ensemble des consommateurs interrogés. A l'opposé, le groupe 2 regroupe une majorité de personnes plus âgées avec $49 \%$ ayant 60 ans et plus contre $37.5 \%$ au total.

Parmi les autres critères permettant d'expliquer le profil des groupes de consommateurs, le groupe 1 est le seul qui regroupe une majorité de femmes avec $58 \%$ contre $40 \% \pm 1 \%$ pour les deux autres groupes. $61 \%$ d'entre eux sont également disposés à payer plus de 21.-

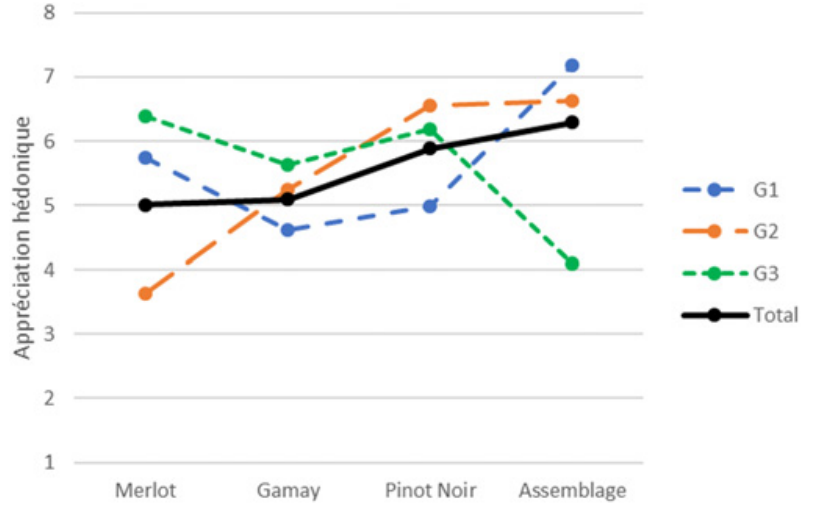

Figure 8. Comparaison des moyennes d'appréciation hédonique des quatre vins rouges pour l'ensemble des 224 consommateurs (trait plein) et pour chacun des trois groupes issus de la segmentation (traits pointillés).

CHF pour une bouteille de vin blanc dans un cadre festif contre seulement $45 \%$ pour les deux autres groupes.

Le groupe 2, au-delà de l'âge plus avancé des consommateurs, peut se caractériser par une majorité de consommateurs provenant de Suisse alémanique (56.6\% contre $45.5 \%$ au total). Si $29 \%$ de l'ensemble des consommateurs disent préférer les vins vaudois, ils ne sont que $17 \%$ à les préférer dans ce groupe. Etonnamment, il s'agit du groupe appréciant plus particulièrement le Chasselas 1993, cépage emblématique du canton de Vaud. Le côté évolué a été jugé positivement par les personnes de ce groupe alors qu'il a été rejeté par les consommateurs réguliers de Chasselas, notamment ceux du groupe 3.

Le groupe 3 est plus hétérogène avec une majorité de 45-59 ans et une part plus importante de personnes originaires du canton de Vaud (41\% contre $33 \%$ dans l'ensemble).

\subsubsection{Vins rouges}

La segmentation des consommateurs en fonction de leurs appréciations hédoniques a également permis de faire ressortir trois groupes distincts. La Fig. 8 montre qu'en vin rouge, la sucrosité ne fait pas autant l'unanimité qu'en vin blanc.

Le groupe 1 est composé de $37 \%$ des consommateurs et regroupe ceux qui apprécient les vins aux structures tanniques plus importantes et particulièrement lorsqu'elles sont associées à du sucre résiduel. Ils préfèrent clairement l'Assemblage avec une moyenne de 7.18 contre 6.29 pour l'ensemble des consommateurs, suivi du Merlot (moyenne de 5.73 contre 5 pour l'ensemble).

Le groupe 2 est composé de $41.5 \%$ des consommateurs qui rejettent le Merlot (moyenne de 3.63 contre 5) et apprécient les vins aux arômes boisés et sucrés (Pinot Noir et Assemblage).

Enfin, le $3^{\text {ème }}$ groupe $(21.5 \%)$ correspond aux consommateurs rejetant l'Assemblage (moyenne de 4.1 contre 6.29 pour l'ensemble des consommateurs) et appréciant le Merlot (moyenne de 6.4).

Les critères permettant de caractériser les consommateurs par groupe sont moins marqués. Le sexe ne caractérise que le $3^{\text {ème }}$ groupe qui rassemble une majorité d'hommes $(66 \%)$ alors que les deux autres groupes sont globalement équilibrés (50\% $\pm 1 \%$ d'hommes). 
Toutefois, les consommateurs du groupe 1 sont ceux achetant les vins rouges les moins chers avec $33 \%$ d'entre eux disant ne pas mettre plus de 20.-CHF par bouteille lors d'une occasion festive (contre seulement $25 \%$ pour l'ensemble des consommateurs).

A l'inverse, $41 \%$ des consommateurs du groupe 2 , se disent prêts à payer plus de $30 .-\mathrm{CHF}$ pour un vin rouge acheté pour une occasion festive.

Enfin, les personnes du groupe 3 sont des consommateurs quotidiens de vin pour $21 \%$ d'entre eux $(12.5 \%$ de consommateurs quotidien au total) et seuls $10 \%$ en consomment seulement mensuellement ou moins $(14.7 \%$ au total).

\section{Discussion et conclusion}

Cette étude montre très nettement que la majorité des consommateurs apprécient des vins contenants entre 5 et $9 \mathrm{~g} / \mathrm{L}$ de sucre résiduel. Toutefois, la sucrosité n'est pas nécessairement perçue de manière nette ou importante mais les vins leurs paraissent plus aromatiques, plus fruités (même pour les vins avec un passage en barrique) et surtout plus équilibrés. Jackson (2009) [9] affirme que $4 \mathrm{~g} / \mathrm{L}$ de sucre résiduel en vin rouge supprime l'acidité, l'astringence et l'amertume. Ces résultats rejoignent les résultats australiens où les vins rouges dépassant 4 à $8 \mathrm{~g} / \mathrm{L}$ de sucre sont relativement courants et particulièrement appréciés [10]. En 2007, Jean-Robert Pitte [7] soulevait le fait que la tendance à faire des vins plus sucrés était déjà présente dans plusieurs vignobles, également en Europe.

Bien qu'ils soient aussi des consommateurs adeptes de boissons sucrées prises au cours des repas, la nouvelle « génération Y» des enfants nés entre 1980 et 2000 est également celle se préoccupant le plus de sa santé, en particulier pour les femmes. La « génération $\mathrm{Z}$ » (nés après 2000) apparait quant à elle comme la première à diminuer sa consommation de soda [11]. Nous avons pu constater que les plus jeunes, notamment les femmes, apprécient également les vins au profil aromatique fruité et ayant une trame acide importante. Selon Pitte (2007) [7], la tendance à plus de sucre dans les vins ne serait très certainement qu'une mode parmi les autres, amenée à disparaitre avec le temps. Avec le recul de la consommation de soda chez les plus jeunes, cette hypothèse peut être maintenue pour les années à venir.

Quant aux vins boisés, souvent considérés comme des vins plus faciles à boire destinés aux jeunes consommateurs, ils se révèlent particulièrement prisés des plus anciens. La baisse de l'acuité sensorielle au cours de la vie a été démontrée dans de nombreuses études. Le vieillissement semble impacter plus fortement la baisse de l'olfaction que la gustation [12]. Cette défaillance du système olfactif explique très certainement leur attirance vers des vins aux profils aromatiques plus prononcés comme le bois ou les arômes d'évolution. Toutefois, leur préférence pour des aliments plus sucrés a également été mise en évidence par plusieurs auteurs $[12,13]$.

La tendance pour des vins plus sucrés, bien que déjà très marquée dans cette étude, pourrait se révéler encore plus probante avec un échantillon de consommateurs moins réguliers de vin. En effet, notre échantillonnage, bien que très important en nombre, sur-représente les consommateurs réguliers de vin par rapport à la population suisse. Selon MIS-TREND (2017) [14], la population suisse compte $28 \%$ de consommateurs occasionnels et $32 \%$ de consommateurs mensuels en 2017, contre seulement $15 \%$ pour le total des deux catégories étudiées.

Enfin, dans une perspective de réchauffement climatique et de concentration plus importante des moûts, l'équilibre entre l'acidité, l'alcool et le sucre devra être judicieusement géré. Maintenir un léger sucre pour ne pas augmenter l'alcool semble être une voie appréciée des consommateurs.

\section{Références}

[1] S. Nicklaus, V. Boggio, S. Issanchou, Arch. Pediatr. 12, 5 (2005)

[2] S.H. Ahmed, K. Guillem, Y. Vandaele, Curr. Opin. Clin. Nutr. Metab. Care 16, 4 (2013)

[3] I.L. Francis, P.O. Williamson, Aust. J. Grape Wine Res. 21 (2015)

[4] F. Filipello, H.W. Berg, E. Hinreiner, A.D. Webb, Food Technol. 9 (1955)

[5] M.A. Amerine, C.S. Ough, Am. J. Enol. Vitic. 18, 3 (1967)

[6] J. Blackman, A. Saliba, L. Schmidtke, Food Qual. Prefer. 21, 7 (2010)

[7] J. Pitte (2007)

[8] F. Boncinelli, L. Casini, C. Contini, F. Gerini, G. Scozzafava, Agric. Agric. Sci. Procedia 8 (2016)

[9] R.S. Jackson, Wine Tasting: A Professional HandBook (Academic Publisher, London, 2009)

[10] P.O. Williamson, J. Robichaud, I.L. Francis, Aust. J. Grape Wine Res. 18, 3 (2012)

[11] B.M. Corporation, 2017. [Online]. Available: https://www . pour-nourrir-demain.fr/cocapepsi-ont-desormais-point-commun-pertejeunes-consommateurs. [Accessed: 30-May2019]

[12] C. Sulmont-Rossé, I. Maître, S. Issanchou, Gérontologie et société 33, 13 (2011)

[13] N. De Jong, C. De Graaf, W.A. Van Staveren, Physiol. Behav. 60, 6 (1996)

[14] MIS TREND (2017) 